\title{
Calorie Restriction for Cancer Prevention and Therapy: Mechanisms, Expectations, and Efficacy
}

\author{
Chiara Vidoni ${ }^{1 *}$, Alessandra Ferraresi ${ }^{1, *}$, Andrea Esposito1, Chinmay Maheshwari', Danny N. Dhanasekaran², \\ Vincenzo Mollace ${ }^{3}$, Ciro Isidoro' \\ ${ }^{1}$ Laboratory of Molecular Pathology, Department of Health Sciences, Università del Piemonte Orientale "A. Avogadro", \\ Novara, Italy, ${ }^{2}$ Stephenson Cancer Center, The University of Oklahoma Health Sciences Center, Oklahoma City, OK, USA, \\ ${ }^{3}$ Department of Health Sciences, Università degli Studi di Catanzaro "Magna Graecia", Catanzaro, Italy
}

\begin{abstract}
Cancer is one of the most frequently diagnosed diseases, and despite the continuous efforts in searching for new and more effective treatments, its morbidity and mortality remain a significant health problem worldwide. Calorie restriction, a dietary manipulation that consists in a reduction of the calorie intake, is gaining attention as a potential adjuvant intervention for preventing and/ or fighting cancer. Several forms of energy reduction intake, which includes caloric restriction tout-court, dietary restrictions, and intermittent fasting, are being explored for their ability to prevent or slow down cancer progression. Additionally, another anti-cancer approach being under investigation relies on the use of nutraceuticals known as "Caloric Restriction Mimetics" that can provide caloric restriction-mediated benefits without subjecting the patients to a strict diet. Preclinical in vitro and in vivo studies consistently show that diet modifiers reducing the calorie have impact on tumor microenvironment and cancer metabolism, resulting in reduced growth and progression of cancer. Preliminary clinical studies show that patients subjected to a reduced nutrient/energy intake experience improved outcomes from chemo- and radiotherapy while better tolerating the side effects. Here, we review the state of the art on the therapeutic potential of calorie restriction and of caloric restriction mimetics in preventing or retarding tumor development by modulating a subset of cellular processes. The most recent clinical progresses with caloric restriction mimetics in the clinical practice are also discussed.
\end{abstract}

Key Words Ketogenic diet, Warburg effect, Caloric restriction mimetics, Tumor microenvironment, Drug therapy

\section{INTRODUCTION}

Calorie restriction (CR) consists in the $20 \%$ to $40 \%$ reduction of the average daily caloric intake without incurring in malnutrition or deprivation of essential nutrients [1]. It encompasses the restriction of specific hypercaloric nutrients which can be substituted with others that are metabolized with less production of energy. Mammals undergo a metabolic adaptation in response to food restriction. The circulating glucose concentration is the first to decline under CR condition, and this results in the utilization of stored glycogen as a main energy source. Once the glycogen stocks are depleted, the organism utilizes glycerol and fatty acids mobilized from the adipose tissue, and thus ketone bodies become the main fuel $[2,3]$.

$\mathrm{CR}$ has a huge impact on promoting longevity by delay- ing the severity and the onset of inflammatory and several age-related diseases including obesity, cardiovascular, neurodegenerative and ophthalmic disorders, and cancer [4]. Such pleiotropic effects rely on several mechanisms, although the principal common denominator is the ability of CR to dampen the oxidative stress and inflammation $[5,6]$.

Aging is one of the major risk factors favoring the development of many cancer types. As a matter of fact, epidemiological studies reveal that five out of six cancer-related deaths occur in patients aged 60 years and older [7]. The continuous exposure to carcinogenic factors, which leads to the accumulation of mutations and epimutations in cancer-sensitive genes surely accounts for the increased risk of cancer development with aging [8]. Additional contributors are the progressive decline of the immune surveillance, the efficien-

Received December 11, 2021, Accepted December 18, 2021

Correspondence to Ciro Isidoro, E-mail: ciro.isidoro@med.uniupo.it, https://orcid.org/0000-0002-5494-3034

Check for updates

*These authors contributed equally to this work as co-first authors. 
cy of DNA repair and of autophagy; the dysregulation of the inflammatory process; the increased production of reactive oxygen species (ROS); increased levels of circulating insulin and many other hormones promoting cell growth, etc. [9-11]. CR may arrest and slow-down age-related decline of cellular protective systems, especially by improving autophagy and dampening inflammation and ROS production [12], as well as reducing circulating growth hormones [13], and this could result in reduced risk of cancer.

Preclinical and preliminary clinical data support the view that reducing calorie intake as well as periodic fasting or dietary restriction (in which intake of macronutrients is limited with no reduction in total calorie) has the potential to prevent and treat cancer $[14,15]$.

Typically, in a CR or dietary restriction regimen, carbohydrates (the main source of energy in the regular diet) are reduced and replaced partially or nearly completely (as in the ketogenic diet) by fat [16]. Indeed, reducing sugar intake seems to be a good strategy to fight cancer, given that cancer cells use glucose as the main fuel $[17,18]$. Likewise providing ketones as alternative energy source may limit cancer growth because cancer cells do not efficiently harness ketones for their anabolism $[19,20]$.

Here, we describe the cellular and molecular mechanisms underlying the pathophysiological effects of calorie and nutrient restrictions and review the scientific proofs of their beneficial effects in preventing cancer onset and progression as well as in improving the anti-cancer therapeutic effects. We also discuss the anti-cancer effects of drugs and nutraceuticals with proven caloric restriction mimetic (CRM) activity. Finally, we present the clinical trials currently investigating the efficacy of caloric restriction dietary regimens as an adjuvant therapy in anti-tumor treatment.

\section{MOLECULAR AND CELLULAR EFFECTS OF CALORIE RESTRICTION AT GLANCE}

For long time, the beneficial impact of $\mathrm{CR}$ was regarded just as a result of the passive effect of nutrient limitation and slow metabolism. It is now recognized that the organismal effects of $\mathrm{CR}$ are actively regulated processes aiming to reduce oxidative stress, and that $C R$ triggers a robust defense program involving multiple metabolic pathways in which nutrient sensors are centrally positioned in such regulation [21]. However, the effects of CR depend on multiple factors such as individual characteristics and the dose and timing of CR [22].

The metabolic adaptations to CR include (i) a decrease in growth factors and production of anabolic hormones [23]; (ii) an upregulation of anti-oxidant systems, which in turn decreases free radical-induced DNA damages [21]; (iii) a downregulation of pro-inflammatory cytokines and an increase in circulating levels of corticosteroids, ghrelin and adiponectin, collectively resulting in the reduction of inflammation [23,24]; and (iv) a delay of aging-associated deterioration of host im- munosurveillance [25]. More in detail, many of the benefits exerted by $\mathrm{CR}$ are associated with the upregulation of genes promoting DNA repair (e.g., genes belonging to the base excision repair pathway), the removal of damaged cells through apoptosis, autophagy, stress response and anti-oxidant defense, in parallel with the downregulation of pro-inflammatory genes and of energy metabolism pathways [23,24,26].

Particularly, autophagy represents the primary stress response to calorie and nutrient restrictions [12]. This process is in fact regulated mainly by two pathways that sense the lack of energy sources and ATP production in the cell, via the AMP-activated kinase (AMPK) and hexokinase 2 (HK2)mTOR complex 1 (mTORC1) pathway, and the lack of growth factors and of amino acids, via the protein kinase $B$ (AKT)-mTORC1 pathway (Fig. 1).

Autophagy (herewith referring to macroautophagy) consists in the p62/SQSTM1-mediated entrapment of cellular components, such as protein aggregates, membranes, and mitochondria (mitophagy) along with portions of cytoplasm, within a double-membrane organelle named autophagosome that upon fusion with the lysosome determines the degradation of those components [27]. This process is regulated by several signaling pathways and autophagy-related (ATG) proteins that also include oncogene products and tumor suppressors, which explains why this process is dysregulated in cancer [28]. Under metabolic stress conditions such as those determined by the lack of nutrients (amino acids, glucose) and of hormones and growth factors, autophagy is upregulated to provide energy and substrates from degradation of redundant self-components [29].

As illustrated in Figure 1, (i) amino acids (especially, methionine, leucine and arginine) directly activate mTORC1 (the mechanistic target of rapamycin complex 1), which then inhibits the axis Unc-51 like autophagy activating kinase 1 complex 1 (ULKC1)-phosphatidylinositol 3-kinase catalytic subunit type 3 (PI3KC3)-BECLIN-1 that positively triggers autophagy; (ii) the presence of growth factors and hormones elicits the activation of mTORC1 via the PI3KC1-AKT pathway thus resulting also in inhibition of autophagy; (iii) soon after entry, glucose is phosphorylated to glucose-6-phosphate (G6P) by HK2, and this prevents HK2 from interacting and inhibiting $\mathrm{mTORC} 1$, and this results in inhibition of autophagy as well. Therefore, autophagy is maximally induced when all these nutrients and growth factors are absent in the tumor microenvironment (TME), as for instance that occurs during starvation. Upregulation of autophagy in cancer cells may have several beneficial outcomes in terms of improved DNA repair efficiency [30], improved TME [31,32], reduced growth and migration/invasive ability [33,34].

\section{CALORIE RESTRICTION AND CANCER PROGRESSION}

From a molecular point of view, several signaling pathways 


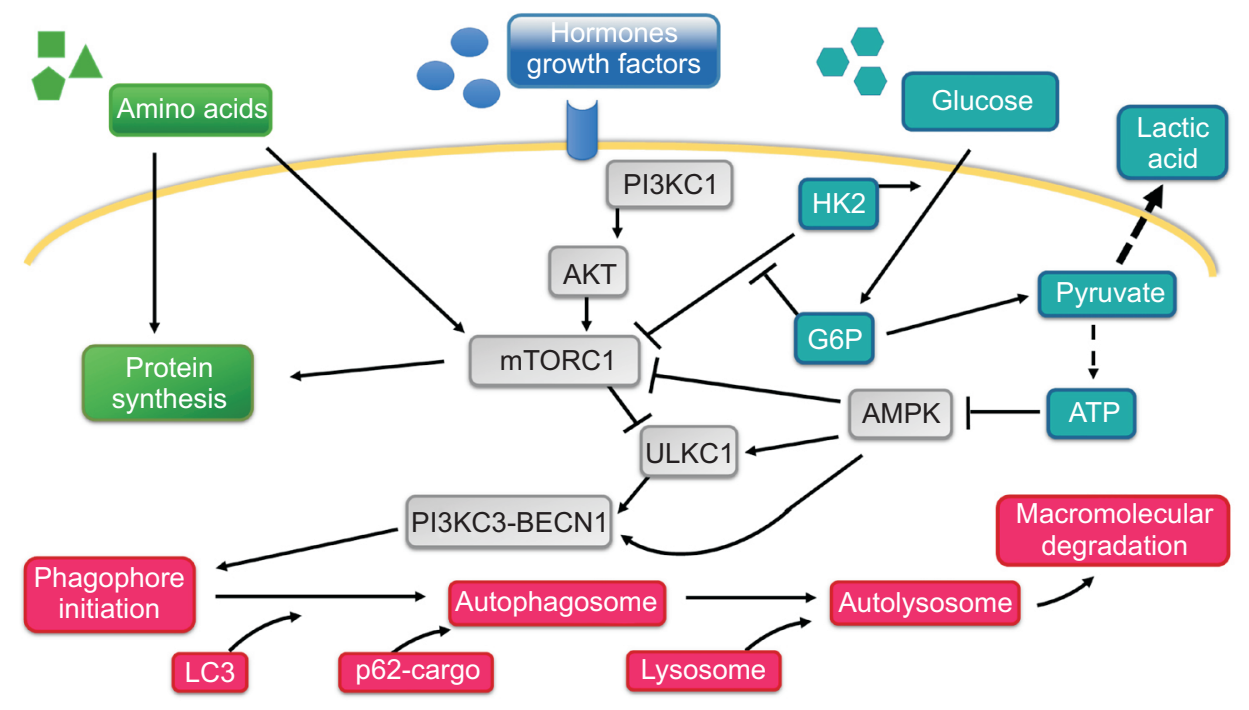

Figure 1. Molecular pathways triggered by caloric restriction at a glance. Caloric restriction impinges on nutrient-sensing pathways to modulate several aspects of cancer cell behavior. Briefly, amino acids availability influences protein synthesis, and hormones and growth factors elicit PI3KC1AKT axis, while glucose intake induces glycolysis that in turn results in lactic acid production. All these pathways cross-talk and converge on mTORC1 that acts as the central hub governing cell metabolism. The latter is a negative regulator of autophagy, a lysosomal-driven catabolic pathway devoted to the macromolecular turnover that is upregulated in response to various cellular stimuli, such as nutrient shortage. PI3KC1, phosphatidylinositol 3-kinase catalytic subunit type 3; HK2, hexokinase 2; AKT, protein kinase B; G6P, glucose-6-phosphate; mTORC1, mTOR complex 1; AMPK, AMP-activated kinase; ULKC1, Unc-51 like autophagy activating kinase 1 complex 1; BECN1, beclin 1; LC3, light chain 3.

collaborate and cross-talk to control carcinogenesis under $\mathrm{CR}$ conditions. To date, the major effectors known to be responsible for the CR-mediated anti-cancer activity include insulin-like growth factor-1 (IGF-1)/phosphatidylinositol-3-kinase (PI3K)/AKT, mTOR, the Sirtuin family proteins, Aldolase A (ALDOA)/DNA-dependent protein kinase (DNA-PK)/p53, NF- $\mathrm{KB}$ and AMPK signaling pathways $[21,35,36]$. However, further studies aiming to characterize the molecular mechanisms by which CR mediates its cancer inhibitory effects are essential for development of new drugs and therapeutic regimens to prevent tumor initiation and/or interrupt tumor promotion and progression. CR can also modulate epigenetic changes, particularly DNA methylation, histone modifications, chromatin remodeling and generation of microRNA, which regulate the expression of genes involved in those processes responsible for $\mathrm{CR}$ anti-cancer activity $[37,38]$.

Notably, CR has been shown to have a wide impact not only on cancer cells but even on TME by allowing enhanced drug delivery, by decreasing the availability of substrate and growth factors for cancer cells, and by reducing inflammation [39-41].

Tumor vascularization represents one of the most crucial steps in cancer progression by ensuring nutrients, soluble factors and oxygen to reach the tumor mass. CR has been capable of counteracting this aspect by hampering the secretion of pro-angiogenic factors such as VEGF, factor VIII, interleukin-6 [IL-6], TNF- $\alpha$, plasminogen activator inhibitor-1 [PAI1], etc. [41-44]. Consequently, tumor neo-vascularization was delayed or even arrested as demonstrated by the reduction in the size, number and density of blood vessels in the CR-fed mice in comparison with the trends observed in ad libitum-fed ones [44,45].

Additionally, CR can shape the tumor immune microenvironment by specifically decreasing the number of tumor associated macrophages, increasing the formation of a reservoir of $\mathrm{CD} 8^{+}$cytotoxic $\mathrm{T}$ cells and memory $\mathrm{T}$ cells while negative modulating immunosuppressive Treg cells' activity and immunosuppressive cytokines levels $[41,42,46,47]$.

Other pivotal players in the TME are the cancer-associated fibroblasts (CAFs), that by releasing oncometabolites, growth factors, inflammatory cytokines and proteolytic enzymes cooperate in the establishment of a malignant liaison between the stroma and cancer parenchymal cells [31]. The evolution of tumor fibrosis, that originates from cancerous lesion, causes an excessive deposition of extracellular matrix and, as a consequence, damaged epithelial cells produce a large amount of pro-inflammatory and pro-fibrotic cytokines, leading to a more and more aggravated deposition of collagen and fibrotic tissue [48]. In this context, CR can elicit an anti-fibrotic effects by downregulating TGF- $\beta$ signaling, that normally promotes the phenotypic conversion of normal fibroblasts in CAFs. In this respect, a highly dense and viscous stroma prevents the cells of the immune system to target the tumor, thus making it much more resistant. By preventing fibrosis, $\mathrm{CR}$ may facilitate the interaction of immune cells with cancer. The remodeling of the TME mediated by CR is schematically 


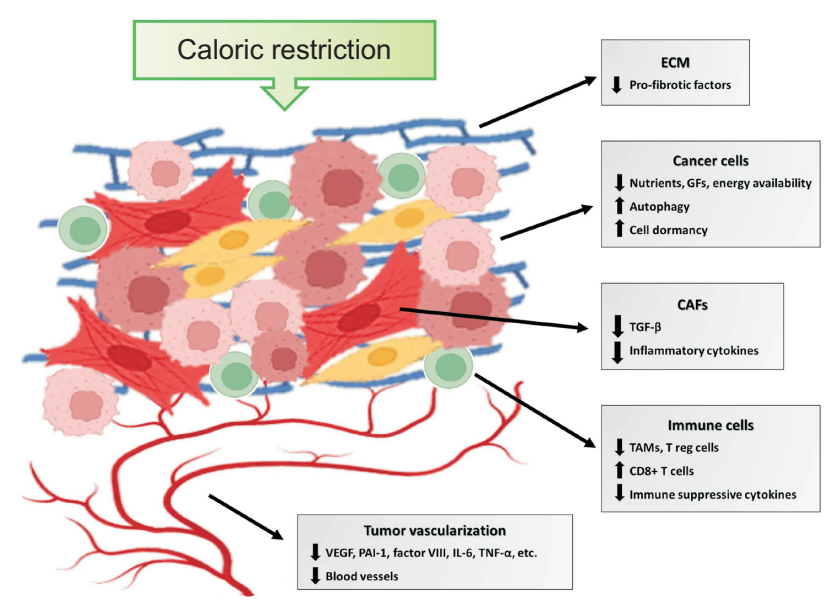

Figure 2. Impact of caloric restriction on the tumor microenvironment. The beneficial effects of caloric restriction are not restricted not only to cancer cells but also involve the other cellular components of the tumor microenvironment. Caloric restriction impinges on ECM remodeling (e.g. by reducing fibrosis), tumor vascularization (e.g. by delaying neo-angiogenesis and decreasing blood vessels density), immune cells (e.g. by counteracting the immune suppressive phenotype) and on CAFs (e.g. by impairing the phenoconversion of normal to activated fibroblasts). ECM, extracellular matrix; GFs, growth factors; CAFs, Cancer-associated fibroblasts; TAMs, tumor associated macrophages; PAI-1, plasminogen activator inhibitor-1; IL-6, interleukin-6.

represented in Figure 2.

\section{BENEFITS OF CALORIC RESTRICTION IN ANTI-CANCER THERAPY}

To date, chemotherapy is one of the main therapeutic strategies for the treatment of several malignancies. However, this approach causes many side effects, such as cardio/neuro/ haematological toxicity, nausea, gastrointestinal symptoms, fatigue, weakness, hair loss and stomatitis, that can negatively affect the cancer patients' quality of life and cause discontinuity of the therapy. Disappointedly, most of the drugs used to manage the symptoms of toxicities may themselves have significant adverse effects.

Although most of the available studies regarding $C R$ in anti-cancer therapy are still in the pre-clinical phase, CR appears a promising approach to modulate the chemotherapy-induced side effects while enhancing the efficacy of the treatment $[40,49,50]$. Reduction of adverse effects would improve quality of life and potentially reduce costs of hospitalization as well as the use of drugs (e.g., anti-emetics, antibiotics, etc.) [51].

In detail, CR can induce healthy cells to invest their energy in reparation and maintenance pathways rather than cell proliferation. This effect promotes an increased resistance of normal cells to chemotherapeutic drugs known as "differential stress resistance". On the other hand, cancer cells bearing mutations in oncogenes (e.g., IGF-1R, Ras, AKT and mTOR pathways, that cause constitutive activation of proliferation pathways in external growth factor-independent manner) and onco suppressor genes (e.g., p53, p16 and Rb, that cause insensitivity to growth-inhibitory signals) are not prone to adapt to fasting conditions and continue to proliferate at a high rate. This results in an enhanced sensitization of cancer cells to chemotherapy-induced apoptosis while protecting normal cells from such effect, leading to the so called "differential stress sensitization" [50-52].

Several reports indicate that fasting potently triggers autophagy, both in normal cells and cancer cells, to recycle critical components and produce energy [50]. The upregulation induction of autophagy before chemotherapy may protect benign cells by providing an alternative mechanism to remove damaged macromolecules and organelles, particularly when the proteasomal degradation pathway is saturated. However, autophagy may also play a pro-survival role in some cancer cells. On the other hand, overactivation of autophagy may lead to what is referred to as autophagy-associated cell death. Given the complex role of autophagy in tumor biology, which is strictly dependent on the context and the stage of malignancy, further studies are needed to dissect the balance between benefits and side effects related to CR-induced upregulation of autophagy $[12,50,53]$.

Even though CR displays numerous benefits in anti-cancer therapy, the real applicability of fasting regimens in the clinical practice could be limited to a small subset of cancer patients, as some potential risks may be associated with this approach, such as malnutrition, cachexia and sarcopenia, that are strongly associated with chemotherapy-related toxicity, reduced response to cancer treatment, low quality of life and a worse overall prognosis [54,55]. Another concern is related to the anti-inflammatory effect of $C R$ that could be disadvantageous for those patients that experience immunodeficiency due to cancer progression and/or as a consequence of repeated chemotherapy treatments [56]. Therefore, more tolerable adjuvant regimens should be developed. In this perspective, fasting-mimicking dietary interventions as well as CRMs (that will be discussed more in detail in the next section) may represent a more feasible therapeutic approach to circumvent these limitations. Overall, the global impact of CR and CRMs on the anti-cancer therapy is illustrated in Figure 3.

\section{CALORIC RESTRICTION MIMETICS}

An alternative therapeutic strategy that extends life expectancy and improves health markers, while reducing the development of several age-related diseases (including cancer), involve use of the pharmacological group of compounds known as CRMs. These compounds act, either through direct interaction with signaling molecules or via epigenetic mechanisms, those pathways that are triggered when energy intake is reduced, yet in the presence of adequate nutrition. 


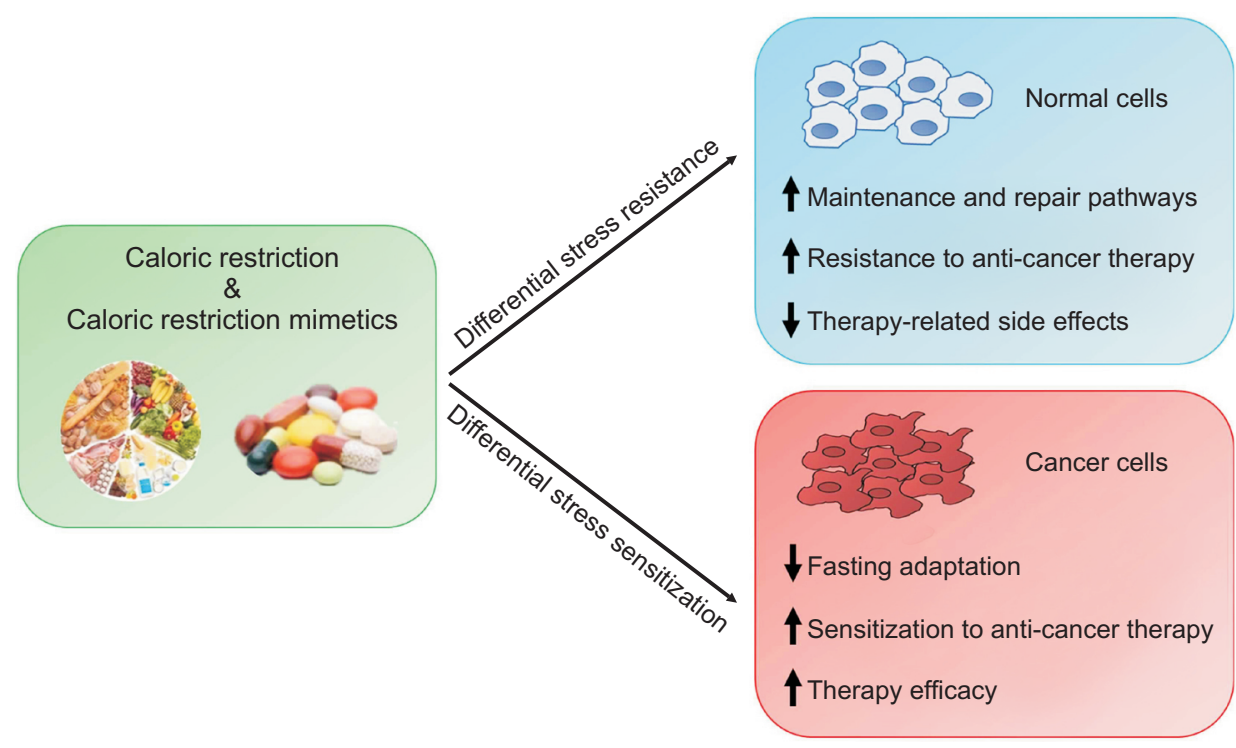

Figure 3. Differential effects of caloric restriction and caloric restriction mimetics on benign and cancer cells. The cartoon compares the differential impact of restriction regimens on normal cells and cancer cells. The differential stress resistance elicited on benign cells is associated with decreased toxicity of therapy and improvement of patients' quality of life, while the differential stress sensitization observed in cancer cells reflects an enhanced efficacy of anti-cancer treatment.
Many CRMs are bioactive food components able to elicit anti-proliferative, pro-apoptotic and anti-metastatic effects [41], avoiding a fasting regimen that could not be tolerated by the cancer patient. The family of polyphenol substances are all a good source of potential CRMs, since they have a wide range of biological activities, including anti-oxidant, anti-inflammatory, anti-carcinogenic and epigenetic modulation activities [57]. They include phenolic acids and derivatives, flavonoids, stilbenes, and coumarins [58].

CRMs modulate energy- and nutrient-sensing pathway impinging on many biological mechanisms, including activation of autophagy, enhancement of insulin sensitivity, inhibition of oxidative stress and inflammation, and modulation of glucose metabolism [59]. The molecular targets of CR involve sirtuins, acetyl-CoA, activated AMP protein kinase, insulin, and mTOR [60].

\section{CRMs in clinical practice}

We will focus on the beneficial effects of the most relevant and promising CRMs, summarized in Table 1, both FDA approved and not yet approved, and will illustrate their potential clinical applications as new effective anti-cancer strategies.

\section{Resveratrol}

Resveratrol (3,5,4-trihydroxystilbene; RV) is a natural stilbene compound presents in vegetables and fruits in general, but especially abundant in grapes [41]. RV acts as a CRM as well as a protein restriction mimetic $[61,62]$. RV has pleiotropic beneficial effects not limited to cancer, but even to metabolic syndromes [63] and neurodegenerative diseases [64]. The tumor suppressive effects of RV on manifestation of malignant phenotype of cancer cells involve the repression of the drug resistance and metastatic ability, counteracting hypoxia, inhibition of inflammation and oxidative stress, etc. [65]. In details, $R V$ reverts cell invasion, which is promoted by high generation of ROS through activation of the Hedgehog pathway $[66,67]$.

Cumulative studies have illustrated the impressive anti-inflammatory properties of RV [68]. In vivo experiments showed that mice treated with RV exhibit low levels of pro-inflammatory cytokines like TNF- $\alpha$, IL-6, IL-1 and IL-8, typical biomarkers of the inflammation [69]. Further, RV increases the number of $\mathrm{T}$ cells, specifically natural killer and $\mathrm{CD} 8^{+} \mathrm{T}$ cytotoxic cells, implementing anti-cancer immunosurveillance [70,71]. Another anti-inflammatory property mediated by RV is the suppression of the NF-kB pathway and of TNF- $\alpha$-induced cancer cell migration and invasion [72]. Additionally, RV can block tumor development by targeting cytochrome $\mathrm{p}-450$ enzymes able to activate pro-carcinogenesis factors [73].

Furthermore, RV positively impacts to expand lifespan as an epigenetic modulator [74], specifically through the activation of sirtuin deacetylases (SIRT1) and autophagy mediated via AMPK pathway [75-77]. Besides limiting glucose uptake and reverting the inflammatory phenotype of CAFs $[78,79]$, $\mathrm{RV}$ is a potent autophagy inducer [77]. Many preclinical and clinical trials in different types of cancer (e.g., breast, colon, and prostate) support its anti-cancer effects [80].

Although RV has many anti-carcinogenic properties, its poor bioavailability limits its clinical use. Nevertheless, there is evidence that RV, either alone or in combination with other agents, is active [81]. Therefore, an alternative strategy is to modify the RV structure for improving its bioavailability and reducing its toxicity [80]. Nowadays, it is clear that RV is a fascinating adjunctive cancer treatment when associated with standard chemotherapeutic agents, but there is still the necessity to define the optimal conditions to ameliorate the delivery and the efficiency [82].

\section{Curcumin}

Curcumin is a polyphenol compound, FDA-approved, for 
Table 1. Overview of the ongoing clinical trials with caloric restriction mimetics (CRMs)

\begin{tabular}{|c|c|c|c|c|}
\hline CRMs & Chemical structure & Cancer type & Metabolic effects & $\begin{array}{c}\text { No. of } \\
\text { clinical trials }\end{array}$ \\
\hline Resveratrol & & $\begin{array}{l}\text { Liver } \\
\text { Colon } \\
\text { Breast } \\
\text { Follicular lymphoma }\end{array}$ & $\begin{array}{l}\text { NAD depletion } \\
\text { Apoptosis } \\
\text { Suppression of } \\
\text { inflammation } \\
\text { Tumor growth } \\
\text { inhibition }\end{array}$ & 17 \\
\hline Curcumin & & $\begin{array}{l}\text { Colorectal } \\
\text { Prostate } \\
\text { Breast }\end{array}$ & $\begin{array}{l}\text { Anti-inflammatory } \\
\text { Anti-oxidant } \\
\text { Anti-angiogenic } \\
\text { Cancer prevention }\end{array}$ & 72 \\
\hline Rapamycin/sirolimus & & $\begin{array}{l}\text { Gynaecological } \\
\text { Breast } \\
\text { Lung } \\
\text { Thyroid }\end{array}$ & $\begin{array}{l}\text { Tumor growth } \\
\text { inhibition } \\
\text { Anti-aging } \\
\text { Anti-inflammatory }\end{array}$ & 765 \\
\hline Everolimus & & $\begin{array}{l}\text { Advanced breast } \\
\text { Renal carcinoma } \\
\text { Intracranial } \\
\text { meningioma }\end{array}$ & $\begin{array}{l}\text { Tumor growth } \\
\text { inhibition } \\
\text { Apoptosis } \\
\text { Anti-inflammatory }\end{array}$ & 621 \\
\hline Metformin & & $\begin{array}{l}\text { Endometrial } \\
\text { Breast } \\
\text { Pancreatic }\end{array}$ & $\begin{array}{l}\text { Anti-inflammatory } \\
\text { Glucose metabolism } \\
\text { reduction } \\
\text { Anti-oxidant }\end{array}$ & 386 \\
\hline Halofuginone & 0 & $\begin{array}{l}\text { Kaposi's sarcoma } \\
\text { Colorectal }\end{array}$ & $\begin{array}{l}\text { Anti-fibrotic } \\
\text { Anti-angiogenic } \\
\text { Apoptosis } \\
\text { Tumor growth } \\
\text { retardation }\end{array}$ & 2 \\
\hline Spermidine & & $\begin{array}{l}\text { Prostate } \\
\text { Skin } \\
\text { Colorectal }\end{array}$ & $\begin{array}{l}\text { Tissue renewal } \\
\text { Cardio-protective } \\
\text { Tumor growth } \\
\text { inhibition }\end{array}$ & 9 \\
\hline
\end{tabular}

The table reports the CRMs employ in ongoing clinical trials in cancer patients. Table elaborated with data extracted from the site https:// clinicaltrials.gov/. 
CRM properties that has caught the attention of many researchers. It is the main bioactive compound isolated from the rhizomes of Curcuma longa (Turmeric) [83]. Several investigations have revealed the multitude of biochemical and biological activities of curcumin with therapeutic potential, including anti-inflammatory, anti-oxidant, anti-cancer and anti-androgenic effects [84].

Particularly remarkable is its anti-cancer activity exerted through induction of apoptosis, inhibition of cell proliferation and of tumor invasion [85], and downregulation of NF- $\kappa B$, COX-2, and STAT3 [84]. Furthermore, curcumin counteracts the Warburg effect (i.e., the aerobic glycolysis occurring in cancer cells) via the suppression of pyruvate kinase M2 (PKM2) [86]. Additionally, curcumin suppresses the PI3K/Akt/ mTOR pathway (by decreasing Akt and mTOR phosphorylation in parallel with PTEN upregulation) thus promoting cell death in cancer cells [87].

Of note, curcumin also abrogates CAF-induced aggressiveness of cancer cells through the inhibition of the mTOR/ HIF-1 $\alpha$ signaling [88]. The anti-carcinogenic property of curcumin is well-documented in several types of cancer, which makes it a promising co-adjuvant agent in cancer therapy [84].

\section{Metformin}

Metformin (dimethylbiguanide hydrochloride) is a derivative of natural biguanidines isolated from the French lilac, Galega officinalis, a plant used for the treatment of type 2 diabetes and metabolic syndrome since 1960's. Metformin administration is not yet certified as adjuvant of anti-cancer therapy [89].

Mechanistically, it suppresses hepatic gluconeogenesis and decreases insulin levels thus acting as a hypoglycemic drug. This effect is attributed to the activation of energy sensor AMPK via the repression of the mitochondrial electron transport chain complex I, thus leading to the inhibition of mTORC1 [90]. For this reason, this molecule is associated with prolonged lifespan, promotion of autophagy, and suppression of oxidative stress and inflammation. As epigenetic modulator, metformin inhibits class II HDACs [91], while stimulates class III HDAC SIRT1 activity [92].

Another important physiological action of metformin involves the immune system. This compound can modulate lymphocyte differentiation during the aging process, promoting $\mathrm{CD}^{+}$memory $\mathrm{T}$ cell differentiation, and simultaneously reducing the expression of several pro-inflammatory cytokines [93]. The latter aspect could represent a relevant opportunity to counteract the development of immune evasion within the TME.

Taken together, metformin has crucial functions in modulating energy metabolism, while its capacity in retarding or contrasting cancer progression is less addressed. In addition, recent clinical trials are also testing its anti-cancer activity, especially in colon, breast, ovarian, prostate and lung tumors [94-97]; however, further investigations are needed.

\section{Spermidine}

Spermidine is a polyamine naturally found in a variety of foods, including wheat germ, soybean, mushrooms, and mature cheese [98]. Further, it is produced by the intestinal microbiota [98].

The activities of this polyamine include the extending lifespan in many model organisms, an effect correlated to induction of autophagy and inhibition of acetyltransferase activity $[99,100]$. Moreover, spermidine stimulates AMPK, while it limits the mTORC1 activity [101]. Predominantly, spermidine is able to stimulate mitophagy in both in vitro [102] and in vivo assays [100], sustaining its capability to slow down aging process and to sustain tissues renewal.

Another molecular mechanism underlying the cancer preventive action of spermidine involves the competition of spermidine with acetyl-CoA for EP300 binding which may contribute to a reduced cancer-related mortality in patients [103]. The inhibition of acetyl transferase EP300 triggers autophagy by the deacetylation of many ATG genes [103]. Furthermore, spermidine, through autophagy activation, can also improve anti-cancer immunosurveillance [104]. To explore and support the spermidine administration as adjuvant anti-cancer treatment, more clinical trials are needed.

\section{Hydroxycitrate}

Hydroxycitrate $(\mathrm{HC})$ or hydroxycitric acid (HCA) is a CRM present in tropical plants as Garcinia cambogia and Hibiscus sabdariffa. It is widely used as a weight-loss drug in obese patients, but it also possesses anti-cancer activity.

A HC's peculiarity is its ability to block acetyl-CoA synthesis by inhibiting the enzyme ATP citrate lyase, thus representing an innovative approach to target cancer metabolism [41]. This compound enhances autophagy flux, since it reduces lysine acetylation of cellular proteins [101]. It has been found that the treatment of $\mathrm{HC}$ promotes the depletion of regulatory $\mathrm{T}$ cells from the tumor, improving immunosuppressive ability and counteracting lung cancer progression [105].

Based on these premises, further synthetic agents, namely acetyl-CoA inhibitors, have been proposed as CRMs: perhexiline maleate is now used in the clinical practice as an anti-anginal agent with cardioprotective and anti-tumor effects [106].

\section{Halofuginone}

Halofuginone (HF) is a synthetic derivative of febrifugine, a natural quinazolinone alkaloid found in the plant Dichroa febrifuga Lour, known for its anti-protozoal activity and used as anti-malarial agent in traditional Chinese medicine [107].

Its ability includes inducing amino acid starvation response (AAR) in cancer cells in parallel with the concomitant activation of autophagy. Accordingly, the molecular explanation is that HF inactivates mTORC1 by causing its detachment from the lysosomes and its degradation in proteasome, while promoting the nuclear translocation of the ATG transcription factor TFEB [108]. 
Furthermore, HF shows its anti-inflammatory propriety by inhibiting the differentiation of inflammatory Th17 cells, an effect clearly linked to induction of AAR [109]. More significantly, $\mathrm{HF}$ is a well-known inhibitor of collagen type I synthesis due to the repression of the TGF- $\beta$ pathway [110]. Further, HF prevents keloid fibrosis by reducing the deposition of ECM and decreasing the proliferation and migration of TGF- $\beta$-activated myofibroblasts [111]. In agreement with this, HF found clinical application as a therapeutic agent in fibrotic disease [112] and in some types of malignancies, such as lung and bladder cancer $[113,114]$. In this respect, more clinical trials are needed to validate the anti-fibrotic property of HF in a wide range of tumors.

\section{Rapamycin}

Rapamycin, also known as sirolimus, is a macrolide compound firstly isolated in 1975 from the bacterium Streptomyces hygroscopicus, found in the soil of Easter Island. Rapamycin is the most promising CRM with an anti-cancer activity, and its efficacy has been addressed in various clinical trials. Its molecular mechanism entails the inhibition of mTOR, a major regulator of cell proliferation and protein synthesis, by binding the protein FKBP12 [115]. Since rapamycin is an inhibitor of mTOR, this CRM promotes autophagy [115]. Consequently, sirolimus provokes the deregulation of mTOR downstream effectors resulting in a prolonged lifespan and in a healthier metabolism [116].

Additionally, this macrolide mediates immunosuppressive effects by controlling survival and proliferation of regulatory T-cells [117]. Because of side effects, including risk of cataract, insulin-resistance and increased infections, it was mandatory to broaden the search for analogues of rapamycin, called rapalogs (e.g., NVP-BEZ235, OSI-027, and RapaLink-1). Everolimus, which belongs to the first-generation rapalogs, was certified for the treatment of hormone receptor-positive, HER2/neu-negative advanced breast cancer [118], whereas temsirolimus (first-generation drug) is identified as a therapeutic agent in metastatic renal cell carcinoma [119]. Therefore, rapamycin's and rapalogs's anti-cancer ability is under investigation in several clinical trials, opening several possibilities for innovative anti-cancer treatments.

To sum up (Table 1), it is well established that CRMs can mimic the actions of $\mathrm{CR}$, or rather delay aging and extend the patients longevity in parallel with improvement of physiological function and reduction of many chronic diseases risk. This results in the avoidance of many side effects occurring with CR together with a better patient's compliance. Nevertheless, even CRMs-based therapeutic approaches show some limitations. For instance, many of them have not been investigated in a sufficient number of clinical trials (e.g., HF, HC, spermidine) in order to guarantee the safety and the feasibility of their applications. Moreover, some CRMs fail to extend lifespan to the same degree as $C R$, suggesting that $C R$ might suppress distinct mechanisms that are partially targeted by
CRMs [57].

Accordingly, innovative clinical protocols for the employment of CRMs are investigated. Recently, the anti-tumor effects of everolimus combined with metformin have been examined. This combination results in an improvement of clonogenicity suppression, cancer cell death and inhibition of mTOR signaling. Therefore, combining different CRMs could synergize their anti-cancer activities in order to achieve health benefits [120].

Additionally, to escalate CRMs effectiveness, it is possible to combine these substances with non-CRM compounds or with nutritional approaches (as $\mathrm{CR}$, intermittent fasting and physical exercise). In this respect, $\mathrm{HC}$, rapamycin and metformin, in association with standard chemotherapeutic drugs, are already applied as anti-cancer therapies [41].

Finally, a great attention is focused on to the relation between CRMs and the "personalized medicine". This results in targeting specific molecular pathways and cancer types with these compounds. As regards, the employment of $\mathrm{HC}$ and spermidine in the fight against lung metastases through the use of aerosolization, an innovative, efficient and non-invasive way to deliver CRMs to the lungs. This method possesses several advantages: first of all, it guarantees a higher local concentration of CRM in a particular tissue and, secondly, it limits the arise of systemic adverse effects [121]. Hence, many researchers have pointed out the existence of specificity of certain CRMs for a precise cancer type. For example, $\mathrm{RV}$ is commonly used in breast cancer therapy in conjunction with chemotherapy [122].

Altogether, new clinical trials need to be undertaken to define how these compounds could become a real "personalized target therapy". By doing that, these mimetics could turn into an effective and adjunctive weapon to fight the battle against cancer.

\section{CONCLUDING REMARKS AND PERSPECTIVES}

Cancer cells are greed of glucose and of amino acids (glutamine, methionine, leucine, arginine, and others) and need growth factors for cell proliferation and cell motility. Thus, starving cancer cells is an appealing strategy to halt cancer growth and metastasization. On this base, it has been hypothesized that a low energetic diet could influence tumor progression and prognosis. Indeed, preclinical and preliminary clinical studies have confirmed that fasting has potential benefits by improving the effectiveness of chemotherapy while attenuating the toxic side effects, by protecting normal tissues from DNA damages, by reducing the inflammation in the TME, by restoring anti-tumor autophagy and apoptosis, and by favoring the immune response [50]. All in all, available data suggest that a regimen with very-low-carbohydrate and low-protein intake, substituted by a relatively high-fat intake, may benefit cancer patients in terms of overall survival and/ 
or progression free survival $[123,124]$. However, patients may not tolerate such a CR diet for prolonged time. Therefore, as alternative, it has been proposed an intermittent fasting regimen, whose beneficial effects also appear promising though somehow controversial in preclinical settings. This will require further elucidation in controlled clinical trials [125]. An interesting alternative is represented by compounds known as CRMs that can mimic the caloric/energic restriction condition while allowing an adequate supplementation of nutrients. These CRMs elicit their action by triggering anti-cancer biochemical pathways through direct interaction with targeted signaling molecules and/or via epigenetic regulation of the expression of relevant regulators. It is likely that CRMs' activity is influenced by the genetic background and the TME context of the tumor. Therefore, understanding the molecular mechanisms underpinning the effects of such CRMs is mandatory for harnessing their adjuvant benefits in the frame of personalized cancer therapy.

\section{ACKNOWLEDGMENTS}

AF is recipient of a post-doctoral fellowship "Paolina Troiano" (id. 24094) granted by Associazione Italiana per la Ricerca sul Cancro (AIRC, Milan, Italy). CV is recipient of a post-doctoral fellowship from Università degli Studi del Piemonte Orientale in collaboration with Università degli Studi Magna Græcia (Catanzaro, Italy). $\mathrm{AE}$ is a $\mathrm{PhD}$ student recipient of a fellowship granted by the Italian Ministry of University and Research (MIUR, Rome, Italy). CM is a PhD student recipient of a fellowship granted by the Italian Ministry of University and Research (MIUR, Rome, Italy) with the contribution of Associazione per la Ricerca Medica Ippocrate-Rhazi (ARMIR, Novara, Italy).

\section{CONFLICTS OF INTEREST}

No potential conflicts of interest were disclosed.

\section{ORCID}

Chiara Vidoni, https://orcid.org/0000-0001-9495-2202

Alessandra Ferraresi, https://orcid.org/0000-0002-7192-9672

Andrea Esposito, https://orcid.org/0000-0002-1214-7312

Chinmay Maheshwari, https://orcid.org/0000-0003-1012-4106

Danny N. Dhanasekaran, https://orcid.org/0000-0001-6350-8926

Vincenzo Mollace, https://orcid.org/0000-0002-0392-7173

Ciro Isidoro, https://orcid.org/0000-0002-5494-3034

\section{REFERENCES}

1. Mitchell SE, Tang Z, Kerbois C, Delville C, Konstantopedos P, Bruel A, et al. The effects of graded levels of calorie restriction: I. impact of short term calorie and protein restriction on body composition in the C57BL/6 mouse. Oncotarget 2015;6:15902-
30.

2. Lee C, Longo VD. Fasting vs dietary restriction in cellular protection and cancer treatment: from model organisms to patients. Oncogene 2011;30:3305-16.

3. Anton SD, Moehl K, Donahoo WT, Marosi K, Lee SA, Mainous AG 3rd, et al. Flipping the metabolic switch: understanding and applying the health benefits of fasting. Obesity (Silver Spring) 2018;26:254-68.

4. Wei M, Brandhorst S, Shelehchi M, Mirzaei H, Cheng CW, Budniak J, et al. Fasting-mimicking diet and markers/risk factors for aging, diabetes, cancer, and cardiovascular disease. Sci Transl Med 2017;9:eaai8700.

5. Fontana L, Partridge L. Promoting health and longevity through diet: from model organisms to humans. Cell 2015;161:106-18.

6. Chung HY, Kim HJ, Kim JW, Yu BP. The inflammation hypothesis of aging: molecular modulation by calorie restriction. Ann NY Acad Sci 2001;928:327-35.

7. Meehan AM, Kassab L, Qin H. Cancer and older adult patient care. Hosp Pract (1995) 2020;48(sup1):17-25.

8. Laconi E, Marongiu F, DeGregori J. Cancer as a disease of old age: changing mutational and microenvironmental landscapes. Br J Cancer 2020;122:943-52.

9. Cha YI, Kim HS. Emerging role of sirtuins on tumorigenesis: possible link between aging and cancer. BMB Rep 2013;46: 429-38.

10. Zinger A, Cho WC, Ben-Yehuda A. Cancer and aging - the inflammatory connection. Aging Dis 2017;8:611-27.

11. Janssen JAMJL. Hyperinsulinemia and its pivotal role in aging, obesity, type 2 diabetes, cardiovascular disease and cancer. Int J Mol Sci 2021;22:7797.

12. Bagherniya M, Butler AE, Barreto GE, Sahebkar A. The effect of fasting or calorie restriction on autophagy induction: a review of the literature. Ageing Res Rev 2018;47:183-97.

13. Salvadori G, Mirisola MG, Longo VD. Intermittent and periodic fasting, hormones, and cancer prevention. Cancers (Basel) 2021;13:4587.

14. Brandhorst S, Longo VD. Fasting and caloric restriction in cancer prevention and treatment. Recent Results Cancer Res 2016;207:241-66.

15. Cadoni E, Marongiu F, Fanti M, Serra M, Laconi E. Caloric restriction delays early phases of carcinogenesis via effects on the tissue microenvironment. Oncotarget 2017;8:36020-32.

16. Klement RJ. Addressing the controversial role of ketogenic diets in cancer treatment. Expert Rev Anticancer Ther 2020;20:32932.

17. Kozal K, Jóźwiak P, Krześlak A. Contemporary perspectives on the Warburg effect inhibition in cancer therapy [published online ahead of print January-December, 2021]. Cancer Control. doi: 10.1177/10732748211041243.

18. Phadngam S, Castiglioni A, Ferraresi A, Morani F, Follo C, Isidoro C. PTEN dephosphorylates AKT to prevent the expression of GLUT1 on plasmamembrane and to limit glucose consumption in cancer cells. Oncotarget 2016;7:84999-5020.

19. Smyl C. Ketogenic diet and cancer-a perspective. Recent 
Results Cancer Res 2016;207:233-40.

20. Lien EC, Vander Heiden MG. A framework for examining how diet impacts tumour metabolism. Nat Rev Cancer 2019;19:65161.

21. Luo H, Chiang HH, Louw M, Susanto A, Chen D. Nutrient sensing and the oxidative stress response. Trends Endocrinol Metab 2017;28:449-60.

22. Green CL, Lamming DW, Fontana L. Molecular mechanisms of dietary restriction promoting health and longevity [published online ahead of print September 13, 2021]. Nat Rev Mol Cell Biol. doi: 10.1038/s41580-021-00411-4.

23. Longo VD, Fontana L. Calorie restriction and cancer prevention: metabolic and molecular mechanisms. Trends Pharmacol Sci 2010;31:89-98.

24. Cangemi A, Fanale D, Rinaldi G, Bazan V, Galvano A, Perez A, et al. Dietary restriction: could it be considered as speed bump on tumor progression road? Tumour Biol 2016;37:7109-18.

25. Messaoudi I, Fischer M, Warner J, Park B, Mattison J, Ingram DK, et al. Optimal window of caloric restriction onset limits its beneficial impact on T-cell senescence in primates. Aging Cell 2008;7:908-19.

26. Gillespie ZE, Pickering J, Eskiw $\mathrm{CH}$. Better living through chemistry: caloric restriction $(\mathrm{CR})$ and $\mathrm{CR}$ mimetics alter genome function to promote increased health and lifespan. Front Genet 2016;7:142.

27. Yang Z, Klionsky DJ. Eaten alive: a history of macroautophagy. Nat Cell Biol 2010;12:814-22.

28. Verma AK, Bharti PS, Rafat S, Bhatt D, Goyal Y, Pandey KK, et al. Autophagy paradox of cancer: role, regulation, and duality. Oxid Med Cell Longev 2021;2021:8832541.

29. Saha S, Panigrahi DP, Patil S, Bhutia SK. Autophagy in health and disease: a comprehensive review. Biomed Pharmacother 2018;104:485-95.

30. Ambrosio S, Majello B. Autophagy roles in genome maintenance. Cancers (Basel) 2020;12:1793.

31. Maes H, Rubio N, Garg AD, Agostinis P. Autophagy: shaping the tumor microenvironment and therapeutic response. Trends Mol Med 2013;19:428-46.

32. Ferraresi A, Girone C, Esposito A, Vidoni C, Vallino L, Secomandi E, et al. How autophagy shapes the tumor microenvironment in ovarian cancer. Front Oncol 2020;10: 599915.

33. Ferraresi A, Phadngam S, Morani F, Galetto A, Alabiso O, Chiorino G, et al. Resveratrol inhibits IL-6-induced ovarian cancer cell migration through epigenetic up-regulation of autophagy. Mol Carcinog 2017;56:1164-81.

34. Ariosa AR, Lahiri V, Lei Y, Yang Y, Yin Z, Zhang Z, et al. A perspective on the role of autophagy in cancer. Biochim Biophys Acta Mol Basis Dis 2021;1867:166262.

35. Lu Y, Tao F, Zhou MT, Tang KF. The signaling pathways that mediate the anti-cancer effects of caloric restriction. Pharmacol Res 2019;141:512-20.

36. Meynet O, Ricci JE. Caloric restriction and cancer: molecular mechanisms and clinical implications. Trends Mol Med 2014;
20:419-27.

37. Khan S, Shukla S, Sinha S, Meeran SM. Epigenetic targets in cancer and aging: dietary and therapeutic interventions. Expert Opin Ther Targets 2016;20:689-703.

38. Li Y, Liu L, Tollefsbol TO. Glucose restriction can extend normal cell lifespan and impair precancerous cell growth through epigenetic control of hTERT and p16 expression. FASEB J 2010;24:1442-53.

39. Kanarek N, Petrova B, Sabatini DM. Dietary modifications for enhanced cancer therapy. Nature 2020;579:507-17.

40. Caccialanza R, Cereda E, De Lorenzo F, Farina G, Pedrazzoli P; AIOM-SINPE-FAVO Working Group. To fast, or not to fast before chemotherapy, that is the question. BMC Cancer 2018;18:337.

41. O'Flanagan $\mathrm{CH}$, Smith LA, McDonell SB, Hursting SD. When less may be more: calorie restriction and response to cancer therapy. BMC Med 2017;15:106.

42. Hursting SD, Dunlap SM, Ford NA, Hursting MJ, Lashinger LM. Calorie restriction and cancer prevention: a mechanistic perspective. Cancer Metab 2013;1:10.

43. Hursting SD, Smith SM, Lashinger LM, Harvey AE, Perkins SN. Calories and carcinogenesis: lessons learned from 30 years of calorie restriction research. Carcinogenesis 2010;31:83-9.

44. Mukherjee P, Abate LE, Seyfried TN. Antiangiogenic and proapoptotic effects of dietary restriction on experimental mouse and human brain tumors. Clin Cancer Res 2004;10:5622-9.

45. Mukherjee P, El-Abbadi MM, Kasperzyk JL, Ranes MK, Seyfried TN. Dietary restriction reduces angiogenesis and growth in an orthotopic mouse brain tumour model. $\mathrm{Br} \mathrm{J}$ Cancer 2002;86:1615-21.

46. Pomatto-Watson LCD, Bodogai M, Bosompra O, Kato J, Wong $\mathrm{S}$, Carpenter M, et al. Daily caloric restriction limits tumor growth more effectively than caloric cycling regardless of dietary composition. Nat Commun 2021;12:6201.

47. Manukian G, Kivolowitz C, DeAngelis T, Shastri AA, Savage $\mathrm{JE}$, Camphausen $\mathrm{K}$, et al. Caloric restriction impairs regulatory $T$ cells within the tumor microenvironment after radiation and primes effector T cells. Int J Radiat Oncol Biol Phys 2021;110:1341-9.

48. Lv XX, Liu SS, Hu ZW. Autophagy-inducing natural compounds: a treasure resource for developing therapeutics against tissue fibrosis. J Asian Nat Prod Res 2017;19:101-8.

49. Lettieri-Barbato D, Aquilano K. Pushing the limits of cancer therapy: the nutrient game. Front Oncol 2018;8:148.

50. Sadeghian M, Rahmani S, Khalesi S, Hejazi E. A review of fasting effects on the response of cancer to chemotherapy. Clin Nutr 2021;40:1669-81.

51. de Groot S, Pijl H, van der Hoeven JJM, Kroep JR. Effects of short-term fasting on cancer treatment. J Exp Clin Cancer Res 2019;38:209.

52. Brandhorst S, Harputlugil E, Mitchell JR, Longo VD. Protective effects of short-term dietary restriction in surgical stress and chemotherapy. Ageing Res Rev 2017;39:68-77.

53. van Niekerk G, Hattingh SM, Engelbrecht AM. Enhanced 
Vidoni et al.

therapeutic efficacy in cancer patients by short-term fasting: the autophagy connection. Front Oncol 2016;6:242.

54. Aapro M, Arends J, Bozzetti F, Fearon K, Grunberg SM, Herrstedt $\mathrm{J}$, et al. Early recognition of malnutrition and cachexia in the cancer patient: a position paper of a European School of Oncology Task Force. Ann Oncol 2014;25:1492-9.

55. Prado CM, Antoun S, Sawyer MB, Baracos VE. Two faces of drug therapy in cancer: drug-related lean tissue loss and its adverse consequences to survival and toxicity. Curr Opin Clin Nutr Metab Care 2011;14:250-4.

56. Ibrahim EM, Al-Foheidi MH, Al-Mansour MM. Energy and caloric restriction, and fasting and cancer: a narrative review. Support Care Cancer 2021;29:2299-304.

57. Madeo F, Carmona-Gutierrez D, Hofer SJ, Kroemer G. Caloric restriction mimetics against age-associated disease: targets, mechanisms, and therapeutic potential. Cell Metab 2019;29:592-610.

58. Singla RK, Dubey AK, Garg A, Sharma RK, Fiorino M, Ameen $\mathrm{SM}$, et al. Natural polyphenols: chemical classification, definition of classes, subcategories, and structures. J AOAC Int 2019;102:1397-400.

59. Yessenkyzy A, Saliev T, Zhanaliyeva M, Masoud AR, Umbayev B, Sergazy S, et al. Polyphenols as caloric-restriction mimetics and autophagy inducers in aging research. Nutrients 2020;12:1344

60. Martel J, Chang SH, Wu CY, Peng HH, Hwang TL, Ko YF, et al. Recent advances in the field of caloric restriction mimetics and anti-aging molecules. Ageing Res Rev 2021;66:101240.

61. Mariño G, Pietrocola F, Madeo F, Kroemer G. Caloric restriction mimetics: natural/physiological pharmacological autophagy inducers. Autophagy 2014;10:1879-82.

62. Omar HA, Berman-Booty L, Kulp SK, Chen CS. Energy restriction as an antitumor target. Future Oncol 2010;6:1675-9.

63. Zeraattalab-Motlagh S, Jayedi A, Shab-Bidar S. The effects of resveratrol supplementation in patients with type 2 diabetes, metabolic syndrome, and nonalcoholic fatty liver disease: an umbrella review of meta-analyses of randomized controlled trials. Am J Clin Nutr 2021;114:1675-85.

64. Vidoni C, Secomandi E, Castiglioni A, Melone MAB, Isidoro C. Resveratrol protects neuronal-like cells expressing mutant Huntingtin from dopamine toxicity by rescuing ATG4-mediated autophagosome formation. Neurochem Int 2018;117:174-87.

65. Han Y, Jo H, Cho JH, Dhanasekaran DN, Song YS. Resveratrol as a tumor-suppressive nutraceutical modulating tumor microenvironment and malignant behaviors of cancer. Int J Mol Sci 2019;20:925.

66. Li W, Cao L, Chen X, Lei J, Ma Q. Resveratrol inhibits hypoxiadriven ROS-induced invasive and migratory ability of pancreatic cancer cells via suppression of the Hedgehog signaling pathway. Oncol Rep 2016;35:1718-26.

67. Ferraresi A, Esposito A, Girone C, Vallino L, Salwa A, Ghezzi I, et al. Resveratrol contrasts LPA-induced ovarian cancer cell migration and platinum resistance by rescuing Hedgehogmediated autophagy. Cells 2021;10:3213.
68. Rahman I, Biswas SK, Kirkham PA. Regulation of inflammation and redox signaling by dietary polyphenols. Biochem Pharmacol 2006;72:1439-52.

69. Timmers S, Konings E, Bilet L, Houtkooper RH, van de Weijer $\mathrm{T}$, Goossens $\mathrm{GH}$, et al. Calorie restriction-like effects of 30 days of resveratrol supplementation on energy metabolism and metabolic profile in obese humans. Cell Metab 2011;14:612-22.

70. Leischner C, Burkard M, Pfeiffer MM, Lauer UM, Busch C, Venturelli S. Nutritional immunology: function of natural killer cells and their modulation by resveratrol for cancer prevention and treatment. Nutr J 2016;15:47.

71. Chen L, Musa AE. Boosting immune system against cancer by resveratrol. Phytother Res 2021;35:5514-26.

72. Ryu J, Ku BM, Lee YK, Jeong JY, Kang S, Choi J, et al. Resveratrol reduces TNF- $\alpha$-induced U373MG human glioma cell invasion through regulating NF- $\mathrm{NB}$ activation and UPA/ uPAR expression. Anticancer Res 2011;31:4223-30.

73. Fouad MA, Agha AM, Merzabani MM, Shouman SA. Resveratrol inhibits proliferation, angiogenesis and induces apoptosis in colon cancer cells: calorie restriction is the force to the cytotoxicity. Hum Exp Toxicol 2013;32:1067-80.

74. Vallino L, Ferraresi A, Vidoni C, Secomandi E, Esposito A, Dhanasekaran DN, et al. Modulation of non-coding RNAs by resveratrol in ovarian cancer cells: In silico analysis and literature review of the anti-cancer pathways involved. J Tradit Complement Med 2020;10:217-29.

75. Pacholec M, Bleasdale JE, Chrunyk B, Cunningham D, Flynn D, Garofalo RS, et al. SRT1720, SRT2183, SRT1460, and resveratrol are not direct activators of SIRT1. J Biol Chem 2010;285:8340-51.

76. Zhao L, Cen F, Tian F, Li MJ, Zhang Q, Shen HY, et al. Combination treatment with quercetin and resveratrol attenuates high fat diet-induced obesity and associated inflammation in rats via the AMPK $\alpha 1 / \mathrm{SIRT} 1$ signaling pathway. Exp Ther Med 2017;14:5942-8.

77. Ferraresi A, Titone R, Follo C, Castiglioni A, Chiorino G, Dhanasekaran DN, et al. The protein restriction mimetic Resveratrol is an autophagy inducer stronger than amino acid starvation in ovarian cancer cells. Mol Carcinog 2017;56:268191.

78. Kueck A, Opipari AW Jr, Griffith KA, Tan L, Choi M, Huang J, et al. Resveratrol inhibits glucose metabolism in human ovarian cancer cells. Gynecol Oncol 2007;107:450-7.

79. Thongchot S, Ferraresi A, Vidoni C, Loilome W, Yongvanit $\mathrm{P}$, Namwat $\mathrm{N}$, et al. Resveratrol interrupts the pro-invasive communication between cancer associated fibroblasts and cholangiocarcinoma cells. Cancer Lett 2018;430:160-71.

80. Singh AP, Singh R, Verma SS, Rai V, Kaschula $\mathrm{CH}$, Maiti $\mathrm{P}$, et al. Health benefits of resveratrol: evidence from clinical studies. Med Res Rev 2019;39:1851-91.

81. Berman AY, Motechin RA, Wiesenfeld MY, Holz MK. The therapeutic potential of resveratrol: a review of clinical trials. NPJ Precis Oncol 2017;1:35.

82. Palminteri M, Dhakar NK, Ferraresi A, Caldera F, Vidoni C, 
Trotta F, et al. Cyclodextrin nanosponge for the GSH-mediated delivery of Resveratrol in human cancer cells. Nanotheranostics 2021;5:197-212.

83. Goel A, Jhurani S, Aggarwal BB. Multi-targeted therapy by curcumin: how spicy is it? Mol Nutr Food Res 2008;52:1010-30.

84. Tomeh MA, Hadianamrei R, Zhao X. A review of curcumin and its derivatives as anticancer agents. Int J Mol Sci 2019;20:1033.

85. Giordano A, Tommonaro G. Curcumin and cancer. Nutrients 2019;11:2376.

86. Siddiqui FA, Prakasam G, Chattopadhyay S, Rehman AU, Padder RA, Ansari MA, et al. Curcumin decreases Warburg effect in cancer cells by down-regulating pyruvate kinase M2 via mTOR-HIF1 $\alpha$ inhibition. Sci Rep 2018;8:8323.

87. Wan Mohd Tajuddin WNB, Lajis NH, Abas F, Othman I, Naidu R. Mechanistic understanding of curcumin's therapeutic effects in lung cancer. Nutrients 2019;11:2989.

88. Du Y, Long Q, Zhang L, Shi Y, Liu X, Li X, et al. Curcumin inhibits cancer-associated fibroblast-driven prostate cancer invasion through MAOA/mTOR/HIF-1 $\alpha$ signaling. Int J Oncol 2015;47:2064-72.

89. Martin-Montalvo A, Mercken EM, Mitchell SJ, Palacios HH, Mote PL, Scheibye-Knudsen M, et al. Metformin improves healthspan and lifespan in mice. Nat Commun 2013;4:2192.

90. Nair V, Sreevalsan S, Basha R, Abdelrahim M, Abudayyeh A, Rodrigues Hoffman A, et al. Mechanism of metformindependent inhibition of mammalian target of rapamycin (mTOR) and Ras activity in pancreatic cancer: role of specificity protein (Sp) transcription factors. J Biol Chem 2014;289:27692-701.

91. Khan S, Jena G. Sodium butyrate reduces insulin-resistance, fat accumulation and dyslipidemia in type-2 diabetic rat: a comparative study with metformin. Chem Biol Interact 2016; 254:124-34.

92. Caton PW, Nayuni NK, Kieswich J, Khan NQ, Yaqoob MM, Corder R. Metformin suppresses hepatic gluconeogenesis through induction of SIRT1 and GCN5. J Endocrinol 2010; 205:97-106.

93. Nath N, Khan M, Paintlia MK, Singh I, Hoda MN, Giri S. Metformin attenuated the autoimmune disease of the central nervous system in animal models of multiple sclerosis. J Immunol 2009;182:8005-14.

94. Kamarudin MNA, Sarker MMR, Zhou JR, Parhar I. Metformin in colorectal cancer: molecular mechanism, preclinical and clinical aspects. J Exp Clin Cancer Res 2019;38:491.

95. De A, Kuppusamy G. Metformin in breast cancer: preclinical and clinical evidence. Curr Probl Cancer 2020;44:100488.

96. Ahn HK, Lee YH, Koo KC. Current status and application of metformin for prostate cancer: a comprehensive review. Int $\mathrm{J}$ Mol Sci 2020;21:8540.

97. Levy A, Doyen J. Metformin for non-small cell lung cancer patients: opportunities and pitfalls. Crit Rev Oncol Hematol 2018;125:41-7.

98. Madeo F, Eisenberg T, Pietrocola F, Kroemer G. Spermidine in health and disease. Science 2018;359:eaan2788.

99. Eisenberg T, Knauer H, Schauer A, Büttner S, Ruckenstuhl
C, Carmona-Gutierrez D, et al. Induction of autophagy by spermidine promotes longevity. Nat Cell Biol 2009;11:1305-14.

100. Eisenberg T, Abdellatif M, Schroeder S, Primessnig U, Stekovic $\mathrm{S}$, Pendl T, et al. Cardioprotection and lifespan extension by the natural polyamine spermidine. Nat Med 2016;22:1428-38.

101. Mariño G, Pietrocola F, Eisenberg T, Kong Y, Malik SA, Andryushkova A, et al. Regulation of autophagy by cytosolic acetyl-coenzyme A. Mol Cell 2014;53:710-25.

102. Qi Y, Qiu Q, Gu X, Tian Y, Zhang Y. ATM mediates spermidineinduced mitophagy via PINK1 and Parkin regulation in human fibroblasts. Sci Rep 2016;6:24700.

103. Pietrocola F, Castoldi F, Kepp O, Carmona-Gutierrez D, Madeo $F$, Kroemer G. Spermidine reduces cancer-related mortality in humans. Autophagy 2019;15:362-5.

104. Pietrocola F, Pol J, Vacchelli E, Rao S, Enot DP, Baracco $E E$, et al. Caloric restriction mimetics enhance anticancer immunosurveillance. Cancer Cell 2016;30:147-60.

105. Rao S, Tortola L, Perlot T, Wirnsberger G, Novatchkova M, Nitsch R, et al. A dual role for autophagy in a murine model of lung cancer. Nat Commun 2014;5:3056.

106. Vella S, Penna I, Longo L, Pioggia G, Garbati P, Florio T, et al. Perhexiline maleate enhances antitumor efficacy of cisplatin in neuroblastoma by inducing over-expression of NDM29 ncRNA. Sci Rep 2015;5:18144.

107. Keller TL, Zocco D, Sundrud MS, Hendrick M, Edenius M, Yum $\mathrm{J}$, et al. Halofuginone and other febrifugine derivatives inhibit prolyl-tRNA synthetase. Nat Chem Biol 2012;8:311-7.

108. Follo C, Vidoni C, Morani F, Ferraresi A, Seca C, Isidoro C. Amino acid response by halofuginone in cancer cells triggers autophagy through proteasome degradation of mTOR. Cell Commun Signal 2019;17:39.

109. Sundrud MS, Koralov SB, Feuerer M, Calado DP, Kozhaya $A E$, Rhule-Smith $A$, et al. Halofuginone inhibits $\mathrm{TH} 17$ cell differentiation by activating the amino acid starvation response. Science 2009;324:1334-8.

110. Shibata A, Kuno M, Adachi R, Sato $Y$, Hattori $H$, Matsuda A, et al. Discovery and pharmacological characterization of a new class of prolyl-tRNA synthetase inhibitor for anti-fibrosis therapy. PLoS One 2017;12:e0186587.

111. Marty $P$, Chatelain $B$, Lihoreau T, Tissot $M$, Dirand $Z$, Humbert $P$, et al. Halofuginone regulates keloid fibroblast fibrotic response to TGF- $\beta$ induction. Biomed Pharmacother 2021;135:111182.

112. Pines M, Nagler A. Halofuginone: a novel antifibrotic therapy. Gen Pharmacol 1998;30:445-50.

113. Li H, Zhang Y, Lan X, Yu J, Yang C, Sun Z, et al. Halofuginone sensitizes lung cancer organoids to cisplatin via suppressing PI3K/AKT and MAPK signaling pathways. Front Cell Dev Biol 2021;9:773048.

114. Elkin M, Ariel I, Miao HQ, Nagler A, Pines M, de-Groot N, et al. Inhibition of bladder carcinoma angiogenesis, stromal support, and tumor growth by halofuginone. Cancer Res 1999;59:4111-8.

115. Boutouja F, Stiehm CM, Platta HW. mTOR: a cellular regulator interface in health and disease. Cells 2019;8:18.

116. Aliper A, Jellen L, Cortese F, Artemov A, Karpinsky-Semper D, 
Moskalev A, et al. Towards natural mimetics of metformin and rapamycin. Aging (Albany NY) 2017;9:2245-68.

117. Strauss L, Czystowska M, Szajnik M, Mandapathil M, Whiteside TL. Differential responses of human regulatory T cells (Treg) and effector T cells to rapamycin. PLoS One 2009;4:e5994.

118. Hasskarl J. Everolimus. Recent Results Cancer Res 2018; 211:101-23.

119. Zanardi E, Verzoni E, Grassi P, Necchi A, Giannatempo P, Raggi $D$, et al. Clinical experience with temsirolimus in the treatment of advanced renal cell carcinoma. Ther Adv Urol 2015;7:152-61.

120. Ariaans G, Jalving M, Vries EG, Jong S. Anti-tumor effects of everolimus and metformin are complementary and glucosedependent in breast cancer cells. BMC Cancer 2017;17:232.

121. Le Noci V, Sommariva M, Bianchi F, Triulzi T, Tagliabue E, Balsari A, et al. Local administration of caloric restriction mimetics to promote the immune control of lung metastases. J Immunol Res 2019;2019:2015892.

122. Casanova F, Quarti J, da Costa DC, Ramos CA, da Silva JL, Fialho E. Resveratrol chemosensitizes breast cancer cells to melphalan by cell cycle arrest. J Cell Biochem 2012;113:258696.

123. Talib WH, Mahmod AI, Kamal A, Rashid HM, Alashqar AMD, Khater S, et al. Ketogenic diet in cancer prevention and therapy: molecular targets and therapeutic opportunities. Curr Issues Mol Biol 2021;43:558-89.

124. Klement RJ, Brehm N, Sweeney RA. Ketogenic diets in medical oncology: a systematic review with focus on clinical outcomes. Med Oncol 2020;37:14.

125. Clifton KK, Ma CX, Fontana L, Peterson LL. Intermittent fasting in the prevention and treatment of cancer. CA Cancer J Clin 2021;71:527-46. 